\title{
Optimal three-ball inequality, quantitative uniqueness for the bi-Laplace equations
}

\author{
Xiaoyu FU ${ }^{1}$ and Zhonghua LIAO $^{1}$ \\ ${ }^{1}$ Sichuan University
}

November 18, 2020

\begin{abstract}
In this paper, we prove an optimal three-ball inequality for bi-Laplace equation in some open, connected set. The derivation of such estimate relies on a delicate Carleman estimate for the bi-Laplace equation and some Caccioppoli inequalities to estimate the lower-ters. Based on three -ball inequality, we then derive the vanishing order of solutions, which is a quantitative version of the strong unique continuation property.
\end{abstract}

\section{Hosted file}

bilaplace1.pdf available at https://authorea.com/users/376959/articles/493773-optimal-threeball-inequality-quantitative-uniqueness-for-the-bi-laplace-equations 